\title{
White matter disruption in autism spectrum disorder is exaggerated by head movements during neuroimaging
}

Diffusion tensor imaging (DTI) studies in children with autism spectrum disorder (ASD) have revealed widespread abnormalities in white matter anatomy, suggesting physical disruption to the brain's key connective structures. In a report recently published in Proceedings of the National Academy of Sciences USA, however, Kami Koldewyn and colleagues argue that the severity of this disruption might have been overestimated owing to artefacts caused by head movement.

\section{4 ...there was no evidence that} ... structural connections are globally affected in ASD... 77

"Several recent papers have shown how head motion during scanning can result in spurious findings in functional connectivity studies," says Koldewyn, "and we wondered if the same might prove to be true in DTI." This problem is expected to be particularly acute in children, as they might struggle to keep still during neuroimaging.

The authors scanned the brains of 52 children with ASD, and compared the results with scans from 73 typically developing children, using a DTI analysis that automatically identified well-validated white matter tracts. When Koldewyn et al. directly compared the two groups of children, the results were consistent with previous findings of global white matter disruption associated with ASD. However, when the children were matched for the amount of head movement observed during scanning, the majority of structural differences vanished.

"After carefully matching the groups for head motion, there was no evidence that long-distance structural connections are globally affected in ASD," explains Koldewyn. Only the right inferior longitudinal fasciculus, which is important for face perception and memory, showed significant structural differences between groups after matching. According to Koldewyn, "if replicated, this finding might be important for understanding the neural and cognitive phenotypes in ASD." Future experiments using similar DTI protocols might also reveal anatomical differences between ASD subgroups.

The implications of this experiment extend beyond the study of child development. The finding that erroneous group differences in brain structure can manifest from motion artefacts underscores the need to properly measure and control for head movements during DTI.

Alex Chase

Original article Koldewyn, K. M. et al. Differences in
the right inferior longitudinal fasciculus but no general
disruption of white matter tracts in children with autism
spectrum disorder. Proc. Natl Acad. Sci. USA doi:10.1073/
pnas.1324037111
the right inferior longitudinal fasciculus but no general spectrum disorder. Proc. Natl Acad. Sci. USA doi:10.1073/ pnas. 1324037111 